\section{Escala numérica para evaluar síntomas espirituales en cuidados paliativos}

\author{
MARÍA MARGARITA REYES ${ }^{a}$, LILIANA DE LIMA ${ }^{\mathrm{b}}$, \\ PAULINA TABOADA ${ }^{1}$, LUIS VILLARROEL ${ }^{\mathrm{c}}$, \\ JUAN DE DIOS VIAL ${ }^{2}$, OSCAR BLANCO ${ }^{d}$, \\ RINA GONZÁLEZ ${ }^{e}$, IRENE PARRA ${ }^{\mathrm{f}}$, GEMITA TOLEDO, \\ PILAR BONATI ${ }^{\text {h }}$, FLAVIO NERVI ${ }^{3}$
}

\section{A scale to assess spiritual symptoms in palliative care}

Background: Spiritual issues are an important dimension of health care, but seldom addressed by professionals. Thus, a scale that assesses the presence and intensity of seven spiritual symptoms was developed. Aim: To validate the instrument in palliative care settings. Material and Methods: The spiritual symptoms scale was applied to 103 patients, aged $59 \pm 17$ years (58\% women), admitted to hospice care in two centers located in Santiago. The reproducibility of the scale was evaluated in 33 patients and its internal consistency and liability in 70. Results: The Fleiss Kappa to assess reproducibility was 0.82 and the analysis of variance had a $p$ of 0.94. Cronbach alpha to assess internal consistency was 0.74. Conclusions: The scale renders similar results when applied by different evaluators and has a good liability. Therefore, it can be a reliable instrument to assess spiritual symptoms in palliative care settings. Further studies would be needed to verify its utility in other settings.

(Rev Med Chile 2017; 145: 747-754)

Key words: Hospice Care; Palliative Care; Personhood; Spirituality; Symptom Assesment.
${ }^{1}$ Médico Internista, PhD en Filosofía, Profesor Asociado, Centro de Bioética, Departamento de Medicina Interna, Facultad de Medicina; Pontificia Universidad Católica de Chile. Santiago, Chile. ${ }^{2}$ Médico; Profesor Titular, Rector Emérito, Pontificia Universidad Católica de Chile. Santiago, Chile. ${ }^{3}$ Médico Gastroenterólogo; Departamento Gastroenterología; Jefe Programa de Medicina Paliativa y Cuidados Continuos; Profesor Titular, Pontificia Universidad Católica de Chile. Santiago, Chile.

aEnfermera, Psicólogo, PhD en Educación; Profesor Asistente Adjunto, Facultad de Medicina, Pontificia Universidad Católica de Chile; Director Ejecutivo Clínica Familia. Santiago, Chile.

besicólogo, Postgrado en Administración de Salud; Director Ejecutivo International.

Association for Hospice and Palliative Care. Houston, Texas, USA.

'Magíster y PhD en Estadística; Profesor Asociado; Departamento de Salud Pública; Facultad de Medicina; Pontificia Universidad Católica de Chile; Santiago, Chile.

'Sacerdote; Clínica Familia; Santiago, Chile. eEnfermera; Magíster en Sociología; Profesora Asociada, Departamento Salud del Niño y Adolescente, Escuela de Enfermería; Pontificia Universidad Católica de Chile. Santiago, Chile. fEnfermera Coordinara, Clínica Familia, Santiago, Chile.

gReligiosa, Enfermera, Clínica Familia, Santiago, Chile.

hEnfermera, Programa de Medicina Paliativa y Cuidados Continuos; Pontificia Universidad Católica de Chile. Santiago, Chile.

Recibido el 8 de noviembre de 2016, aceptado el 3 de julio de 2017.

Financiamiento: no.

Correspondencia a:

María Margarita Reyes

Avda. Antonio Varas 1779, Providencia.

Santiago, Chile.

mmreyesdo@gmail.com
L a experiencia clínica y la literatura avalan que la espiritualidad guía el comportamiento de las personas en relación a la vivencia de su enfermedad y a la toma de decisiones en salud, tanto en los cuidados físicos, como en los psicológicos ${ }^{1-4}$. Es habitual que los pacientes necesiten explorar, compartir y encontrar significado a la enfermedad y a su muerte próxima ${ }^{5}$. En la literatura se describe una correlación inversa entre el bienestar espiritual y la frecuencia de depresión clínica, desesperanza y deseo de morir ${ }^{6,7}$.
La importancia que debería tener la dimensión espiritual en la atención sanitaria se ve claramente reflejada en la definición de cuidados paliativos que propuso la Organización Mundial de Salud (OMS) en 1990, como "el cuidado activo $y$ total de los pacientes portadores de enfermedades que no responden a tratamiento curativo [enfatizando que] el control del dolor y de otros síntomas, así como la atención de aspectos psicológicos, sociales y espirituales, es primordial". Este énfasis se reitera en la definición de $2002^{9}$. Así, en cuidados paliativos tradicionalmente ha 
existido una mirada integral del ser humano, que abarca las dimensiones espiritual, psicosocial y física $^{10,11}$.

La necesidad de integrar la espiritualidad no sólo en los cuidados paliativos, sino también en todos los niveles de la atención sanitaria, ha sido estudiada en las últimas décadas por el equipo de la Dra. Cristina Puchalski, del George Washington University's Institute for Spirituality and Health (GWish), que además ha desarrollado estrategias docentes orientadas al "cuidado total" de los pacientes $^{12-18}$. Otros aportes significativos en este mismo ámbito han hecho la European Association of Palliative Care (EACP) ${ }^{19}$ y el Grupo de Espiritualidad de la Sociedad Española de Cuidados Paliativos (SECPAL), liderado por el Dr. Enric Benito. Diversos cuestionarios breves para evaluar la dimensión espiritual durante la entrevista clínica han sido elaborados ${ }^{20,21}$.

En Chile, la Ley 20.584, que Regula los Derechos y Deberes que tienen las Personas en relación con Acciones Vinculadas a su Atención en Salud ${ }^{22}$ establece que "toda persona que lo solicite tiene derecho a recibir, oportunamente y en conformidad a la ley, consejería y asistencia religiosa o espiritual". (art. 6).

Sin embargo, en nuestro país el abordaje de los aspectos espirituales en la práctica clínica es limitado. Las dificultades comienzan desde la misma definición. ¿Qué se entiende por espiritualidad en salud? En el 2011, la EAPC definió espiritualidad como "la dimensión dinámica de la vida humana que se relaciona con el modo en que las personas (individuo y comunidad) experimentan, expresan y/o buscan significado, propósito y trascendencia, y el modo de conectarse con el momento, el 'yo', los otros, la naturaleza, lo significativo y/o lo sacro" ${ }^{19}$. Esta definición recoge muy bien los conceptos elaborados previamente por diversos autore ${ }^{23-24}$ y coincide con la definición de consenso propuesta recientemente en los Estados Unidos de Norteamérica, que agrega que la espiritualidad se expresa a través de creencias, valores, tradiciones y prácticas ${ }^{12}$.

Para efectos de este trabajo, entenderemos por espiritualidad la dimensión dinámica y fundamental en la vida del ser humano y su entorno, que se refiere a los valores, los aspectos éticos, la búsqueda de significado y la trascendencia.

Diversos autores han propuesto instrumentos para evaluar, en forma lo más objetiva posible, la dimensión espiritual en la práctica clínica ${ }^{25-27}$. Entre los sistemas de evaluación descritos en la literatura se encuentran instrumentos que miden distintas expresiones de la dimensión espiritual ${ }^{28}$, como p. ej. 1) La orientación religiosa; 2) La historia espiritual; 3) Las "necesidades espirituales"; 4) El "bienestar espiritual"29; 5) Los problemas espirituales $^{23}$ y 6 ) "Distress espiritual".

Entre los problemas espirituales, Speck describe: 1) culpabilidad; 2) desconfianza; 3 ) búsqueda espiritual; 4) angustia espiritual; 5) desesperanza; 6) dificultad en el desapego y 7) falta de fe ${ }^{23}$.

Nuestra hipótesis es que los así llamados "problemas espirituales" pueden expresarse a través de "sintomas espirituales", factibles de ser identificados y cuantificados de un modo estadísticamente confiable. Esta hipótesis plantea el desafío de crear y validar un instrumento adecuado ${ }^{30-33}$ que además sea aplicable a nuestra realidad latinoamericana. Debido a que los instrumentos de evaluación en espiritualidad descritos en la literatura han sido desarrollados y validados en Norteamérica y Europa Occidental, se planteaban inconvenientes para su aplicación en pacientes de otras regiones del mundo.

\section{Material y Método}

\section{Diseño}

Estudio observacional, analítico y de corte transversal, con metodología cuantitativa, que incluyó dos pruebas de validación de la Escala Numérica para Evaluar Síntomas Espirituales (ENESE): la primera para medir reproducibilidad y detectar errores de redacción e interpretación, con el fin de obtener un instrumento mejorado; la segunda para evaluar la confiabilidad o consistencia interna del instrumento y su validez de constructo a través del análisis factorial. El ENESE fue aplicado por tres enfermeras de Cuidados Paliativos especialmente entrenadas para ello, contando además con un instructivo elaborado por los Investigadores Responsables. Las entrevistas se realizaron entre junio de 2009 y junio de 2010.

El estudio fue aprobado por el Comité Ético-Científico de la Facultad de Medicina de la Pontificia Universidad Católica de Chile (02/06/2009).

Los pacientes fueron invitados a participar libremente, informados sobre los objetivos del estudio y firmaron un documento de Consentimiento Informado antes de su participación ${ }^{34}$. 


\section{Participantes}

La muestra incluyó 103 pacientes hospitalizados en dos centros de cuidados paliativos de Santiago de Chile: la Clínica Familia y el Hospital Clínico de la Universidad Católica.

Los criterios de inclusión fueron:

- Diagnóstico de enfermedad terminal, certificada por un médico.

- Paciente en conocimiento de su diagnóstico y pronóstico.

- Presencia de algún "problema espiritual".

- Ausencia de alteraciones o presencia de alteraciones leves en el test de CAM (Confusion Assessment Method) y en el Mini Mental Abreviado ${ }^{35,36}$.

\section{Instrumentos de medida}

- Prueba 1 (reproducibilidad del instrumento): El ENESE se aplicó inicialmente a una muestra de 33 pacientes, con el objetivo de evaluar la comprensibilidad y reproducibilidad del instrumento al ser aplicado por tres enfermeras a un mismo paciente. Esta prueba permitió reformular las preguntas en cuatro de los siete síntomas y obtener un instrumento mejorado, con una definición estandarizada para cada síntoma (Tabla 1).
- Prueba 2 (consistencia interna del instrumento $y$ validez de constructo): El instrumento mejorado (Tabla 2) se aplicó a una muestra de 70 pacientes, para evaluar su consistencia interna y validez de constructo. El tamaño muestral para esta prueba se determinó mediante la fórmula clásica de Freeman ${ }^{37}$, según el número de preguntas o ítems a evaluar.

\section{Análisis estadístico}

Para la Prueba 1, que medía la reproducibilidad del instrumento, se usó el Índice de Kappa de Fleiss $(\mathrm{K})$ y análisis de varianza para medidas repetidas (ANOVA).

Para la Prueba 2, que evaluaba la consistencia interna del instrumento, se utilizó el test de Alfa de Cronbach ${ }^{38-40}$. La confiabilidad se establece con un índice sobre 0,7 . Para evaluar la validez de constructo se utilizó análisis factorial con rotación Varimax, para determinar la existencia de posibles sub-escalas al interior del instrumento. Se usó el test de $\chi^{2}$ para comparar proporciones entre sub-grupos. Se consideró significativo todo valor $\mathrm{p}$ inferior o igual a 0,05 . El análisis de datos se hizo mediante el programa estadístico SPSS 17.0 para Windows.

Tabla 1. Definición de síntomas espirituales para ENESE

1. Dolor espiritual: Sensación de infelicidad o desagrado interior por la situación existencial de terminalidad. El paciente puede referir que esta sensación displacentera o estado de infelicidad está asociada a la incapacidad de satisfacer necesidades y/o aspiraciones personales. Puede acompañarse de otros síntomas espirituales (ej. angustia, desesperación) y otros síntomas físicos y/o psíquicos confusos

2. Auto-castigo: Manifestación de daño causado a sí mismo, en forma consciente o inconsciente, por un sentimiento de culpa relacionado con faltas no perdonadas (por sí mismo, por los demás o por su creencia) y/o no reparadas. Puede asociarse a angustia y/o sufrimiento

3. Búsqueda espiritual proyectada: Expresión de la necesidad espiritual no reconocida conscientemente por el paciente, que se proyecta en otra persona y/o síntoma. Puede sospecharse cuando los síntomas físicos no son explicables y/o no responden a las intervenciones habituales o cuando se plantean exigencias persistentes a la familia o al personal. Suele generar angustia, inquietud y sufrimiento en el enfermo e impotencia en los familiares y/o el personal

4. Angustia espiritual: Aflicción incontrolable relacionada con la percepción de significado o sentido negativo atribuido a la propia historia de vida, la experiencia de enfermedad y la muerte próxima

5. Incredulidad: Ausencia de fe en sí mismo, en los demás y/o en su creencia. Suele expresarse como dificultad para acoger la propia realidad existencial, desconfianza y/o falta de apoyo en su creencia (religiosa u otra)

6. Desesperanza: Percepción de imposibilidad de obtener lo que se desea ("un bien futuro") y/o de satisfacer sus necesidades. Puede acompañarse de aflicción ante un peligro real, inevitable, incontrolable y desconocido. En ocasiones se expresa con actitudes hostiles

7. Desamor: Sensación de indiferencia, molestia o rabia hacia sí mismo, hacia otras personas y/o hacia a su creencia. Puede acompañarse de insatisfacción, sensación de vacío interior y/o desvalorización 


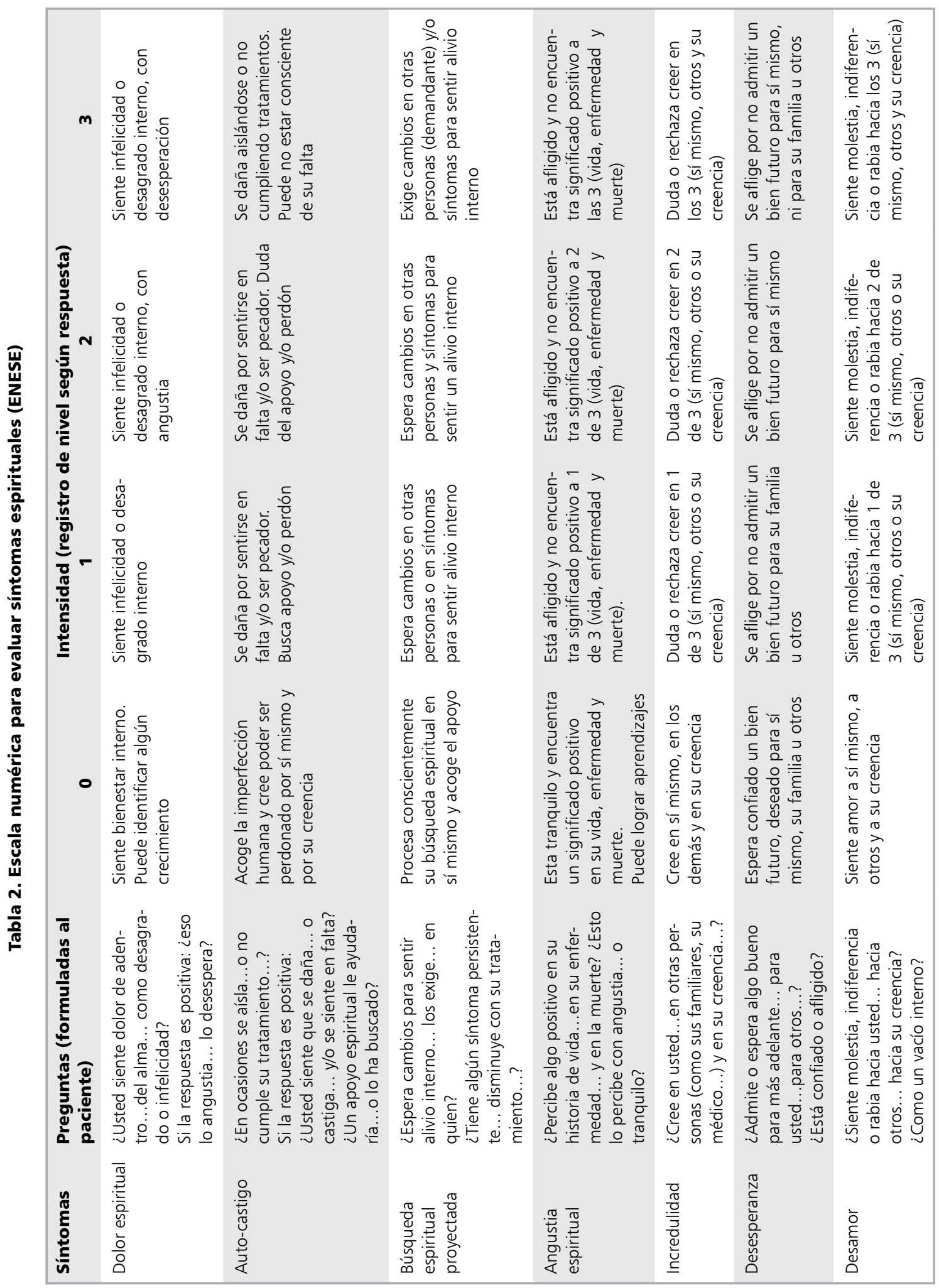




\section{Resultados}

De la muestra estudiada, $58 \%$ eran de sexo femenino, con una edad promedio de 58,7 años (desviación estándar: $\pm 16,8$; rango: $31-97$ años).

En la Tabla 3 se muestran la frecuencia y el porcentaje de sintomas espirituales detectados mediante ENESE. Destaca la alta frecuencia de sintomas espirituales, siendo la presencia de 'angustia espiritual' el síntoma más frecuente $(91,4 \%)$, seguido por el 'dolor espiritual' y la 'búsqueda espiritual proyectada' (el $88,8 \%$ y el $85,7 \%$ respectivamente). La 'incredulidad' fue el síntoma menos frecuente $(67,1 \%)$.

Tabla 3. Frecuencia y porcentaje de síntomas espirituales

\begin{tabular}{|lrrrr|}
\hline Síntoma & \multicolumn{2}{c}{ Ausencia } & \multicolumn{2}{c|}{ Presencia* } \\
& n & \% & \multicolumn{1}{c|}{$\mathbf{n}$} & \% \\
\hline Dolor espiritual & 8 & 11,4 & 62 & 88,6 \\
\hline Auto-castigo & 16 & 22,9 & 54 & 77,1 \\
\hline $\begin{array}{l}\text { Proyección búsqueda } \\
\text { espiritual }\end{array}$ & 10 & 14,3 & 60 & 85,7 \\
\hline Angustia espiritual & 6 & 8,6 & 64 & 91,4 \\
\hline Incredulidad & 23 & 32,9 & 47 & 67,1 \\
\hline Desesperanza & 21 & 30,0 & 49 & 70,0 \\
\hline Desamor & 16 & 22,9 & 54 & 77,1 \\
\hline
\end{tabular}

*Presencia incluye la evaluación positiva en cualquiera de las categorías $(1,2,3)$ del ENESE.

\section{Prueba 1 (reproducibilidad del instrumento)}

Durante la aplicación inicial del instrumento (33 pacientes) se detectaron algunos problemas menores de redacción, que dificultaban su comprensión por parte de los pacientes. De este modo, se reformularon las preguntas para cuatro de los siete síntomas.

El análisis de reproducibilidad del instrumento, medida a través del Índice Kappa de Fleiss fue 0,817 y el análisis de la varianza para medidas repetidas, arrojó un valor $\mathrm{p}=0,94$, que se estima altamente significativo. Esto quiere decir que el instrumento (ENESE) puede ser aplicado por distintos evaluadores pudiendo esperarse resultados similares entre ellos.

\section{Prueba 2 (confiabilidad y consistencia interna del instrumento)}

El análisis de la confiabilidad y consistencia interna del instrumento aplicado a los 70 pacientes se hizo mediante el test Alfa de Cronbach, que resultó igual a 0,74 . Esto significa que la escala estudiada es confiable ${ }^{38,39,40}$. En la Tabla 4 se observa una descripción del comportamiento que tendría la escala si se eliminara cualquiera de los síntomas en estudio. Se observa que el dolor espiritual es el síntoma de mayor influencia en la fiabilidad del ENESE, ya que, si se eliminara este síntoma, la confiabilidad disminuiría a 0,671. Si se eliminara cualquiera de los otros síntomas la escala no sufriría grandes modificaciones.

En la validez de constructo, el análisis factorial identificó dos sub-grupos de síntomas al interior

Tabla 4. Resultados análisis estadístico de fiabilidad por elemento o variable

\begin{tabular}{|lcccc|}
\hline Elemento o variable & $\begin{array}{c}\text { Media de la escala } \\
\text { si se elimina el } \\
\text { elemento }\end{array}$ & $\begin{array}{c}\text { Varianza de la } \\
\text { escala si se elimina } \\
\text { el elemento }\end{array}$ & $\begin{array}{c}\text { Correlación } \\
\text { elemento-total } \\
\text { corregida }\end{array}$ & $\begin{array}{c}\text { Alfa de Cronbach } \\
\text { si se elimina el } \\
\text { elemento }\end{array}$ \\
\hline $\begin{array}{l}\text { Dolor espiritual } \\
\text { Auto castigo }\end{array}$ & 9,34 & 13,968 & 0,591 & 0,671 \\
\hline $\begin{array}{l}\text { Proyección búsqueda } \\
\text { espiritual }\end{array}$ & 9,64 & 14,262 & 0,428 & 0,714 \\
\hline Angustia espiritual & 9,76 & 16,708 & 0,246 & 0,749 \\
\hline Incredulidad & 9,70 & 15,662 & 0,470 & 0,703 \\
\hline Desesperanza & 10,41 & 15,782 & 0,409 & 0,715 \\
\hline Desamor & 10,21 & 14,635 & 0,502 & 0,694 \\
\hline
\end{tabular}


del instrumento, que explican en su conjunto el 55\% de la varianza de los datos: 1) La 'proyección de la búsqueda espiritual' con la 'desesperanza'; y 2) los cinco síntomas restantes.

Como hallazgo del estudio, se constató una asociación espontánea que hicieron los pacientes entre algunos de los síntomas y ciertas expresiones, que se repitieron. Así, al evaluar el 'dolor espiritual' manifestaban que "el dolor ayuda a crecer" y en el caso del 'desamor', expresaban una "sensación de vacío interno”. El test de $\chi^{2}$ mostró una diferencia significativa en dichas asociaciones para ambos síntomas $(p=0,001)$. En cambio, para la 'angustia espiritual', que se asoció a la expresión "ayuda a aprender", la diferencia no fue significativa $(\mathrm{p}=0,06)$.

\section{Discusión}

Los resultados de este estudio muestran la fiabilidad y validez del instrumento utilizado (ENESE) para evaluar la presencia e intensidad de siete síntomas espirituales en pacientes hospitalizados en dos centros de cuidados paliativos en Santiago de Chile. Se verifica que el instrumento puede ser aplicado por evaluadores diferentes con resultados muy similares. De este modo, se confirma la hipótesis de este estudio, que la presencia e intensidad de los sintomas espirituales en los pacientes en cuidados paliativos pueden ser cuantificados de un modo estadísticamente confiable.

Además, como un hallazgo interesante del estudio, se constató que los pacientes tendían a asociar dos de los síntomas evaluados: la 'búsqueda espiritual proyectada' y la 'desesperanza'. Una posible explicación de este hallazgo sería que con frecuencia la 'búsqueda espiritual proyectada' no es reconocida como tal por el propio enfermo, lo que podría aumentar la expresión del síntoma 'desesperanza'.

Por otro lado, la asociación espontánea que los pacientes hicieron de algunos de los siete síntomas con ciertas expresiones (como p.ej.: "el dolor espiritual ayuda a crecer"; "la angustia espiritual ayuda a aprender"; el 'desamor' expresado como "sentimiento de vacío interno”), podría interpretarse como un reflejo de las diferentes percepciones que tiene cada individuo de su espiritualidad. Ello sugiere la necesidad de conocer mejor el signifi- cado que los pacientes en Cuidados Paliativos le atribuyen a su espiritualidad, aspecto que podría investigarse en el futuro.

Dentro de las limitaciones de este estudio cabe señalar que fue realizado en Chile, por lo que sus resultados con pacientes de otras culturas y lenguajes pueden ser diferentes. La mayoría de la población chilena es católica $y$, aunque este estudio no está dirigido a las creencias religiosas específicas, los síntomas espirituales pueden variar en poblaciones en las cuales hay predominio de otras creencias y afiliaciones religiosas. Además, el diseño de este estudio no permite medir la especificidad del instrumento (ENESE), pues uno de los criterios de inclusión de los pacientes fue la presencia de "problemas espirituales", lo que podría relacionarse con la alta frecuencia encontrada de "sintomas espirituales" (ENESE). Por tanto, no es posible predecir lo que pasaría si se aplica este instrumento a pacientes que no presenten "problemas espirituales". Sin embargo, este escenario es difícil de imaginar, especialmente en pacientes de Cuidados Paliativos.

Por otra parte, en este estudio no se correlacionó el ENESE con otros instrumentos, ni con otras variables de la atención de salud, como días de hospitalización o calidad de vida. Ello debido a que en la literatura revisada no se encontraron otras escalas que evaluaran sintomas espirituales. Esto plantea un interesante desafío para investigaciones futuras.

Contar con un instrumento validado para evaluar la presencia e intensidad de sintomas espirituales en pacientes en Cuidados Paliativos puede contribuir a su mejor atención, incluyendo la espiritualidad como una dimensión central del cuidado integral. La posibilidad de detectar la presencia de sintomas espirituales sugiere la necesidad de elaborar estrategias adecuadas para su intervención, con el fin de contribuir al bienestar espiritual del paciente.

Por otro lado, disponer de un instrumento validado para evaluar la dimensión espiritual, además de ayudar al equipo de salud a tener un lenguaje común, puede facilitar la docencia y el aprendizaje del cuidado integral de los pacientes al final de la vida.

Por ahora, el ENESE está disponible solamente en español y ha sido validado en Chile, con pacientes adultos de Cuidados Paliativos. Será necesario realizar otros estudios para medir la aplicabilidad 
de ENESE en otro tipo de pacientes (ej. crónicos, pediátricos, geriátricos, etc.), en otros países, y en otros idiomas.

Agradecimientos: Los autores valoran y agradecen especialmente la participación de los pacientes en este estudio, así como los consejos de los siguientes expertos: José Carlos Bermejo, María Ignacia del Río y Gloria Uribe.

\section{Referencias}

1. Rousseau P. The art of oncology: When the tumor is not target. Spirituality and the Dying Patient. Clinical Oncology 2000; 18 (9): 2000-2.

2. Ming-Shium T. Illnes: An Opportunity for Spiritual Growth. The Journal of Alternative and Complementary Medicine 2006; 12 (10): 1029-33.

3. Reyes M. Formación en valores a familias de enfermos terminales. Tesis Doctoral. Sevilla, España: Universidad de Sevilla; 2003.

4. Freud S. Esquema del psicoanálisis (1937-1939). Doctrina de las pulsiones (1938). O.C. XXIII, En: Freud S. Obras Completas. Buenos Aires, Argentina. Amorrortu Editores. 1976.

5. Benedicto XVI. Caritas in Veritate. Città del Vaticano: Libreria Editrice Vaticana; 2009.

6. Harvey BM. Interventions to Enhance the Spiritual Aspects of Dying. Journal of Palliative Medicine 2005; 8: 103-15.

7. Nelson CR, Breitbart W, Galieta M. Spirituality, Religion and Depression in the Terminally III. Psychosomatics 2002; 43 (3): 213-20.

8. Organización Mundial de la Salud (OMS). Alivio del dolor y tratamiento paliativo en cáncer. Informe de un Comité de expertos. Organización Mundial de la Salud, Serie de Informes Técnicos 804: Ginebra, Suiza: 1990, p. 11-2.

9. World Health Organization (WHO). National Cancer Control Programmer: Policies and Managerial Guidelines, 2nd Ed. Geneva, Switzerland: WHO, 2002.

10. Doyle D, Hanks D, MacDonald M. Introduction. En: Doyle D, Hanks D, MacDonald M. Oxford Textbook of Palliative Medicine. Oxford, U.K.: Oxford University Press. 2003; p. 3-11.

11. Doyle D, Hanks D, MacDonald M. The emotional problems of the patient. En: Doyle D, Hanks D, MacDonald M. Oxford Textbook of Palliative Medicine. Oxford, U.K.: Oxford University Press. 2003. p. 903.

12. Puchalski C, Vitillo R, Hull S and Reller N. Improving the Spiritual Dimension of Whole Person Care: Reaching National and International Consensus. Journal of Palliative Medicine 2014; 17 (6): 1-9.

13. Puchalski C, Blatt B, Kogan M, Butler A. Spirituality and health: The development of a field. Acad Med 2014; 89: 10-6.

14. Cobb M, Puchalski C, Rumbold B. Oxford Textbook of Spirituality in Healthcare. Oxford, U.K.: Oxford University Press. 2012.

15. Puchalski C, Ferrell B, Virani R, Otis-Green S, Baird $\mathrm{P}$, Bull J, et al. Improving the Quality of Spiritual Care as a Dimension of Palliative Care: The Report of the Consensus Conference. Journal of Palliative Medicine. October 2009: 885-904.

16. Puchalski C. Spirituality and medicine: curricula in medical education. Cancer Educ 2006; 21 (1): 14-8.

17. Puchalski C. A Time for Listening and Caring. Spirituality and Care for the Chronically Ill and Dying. Oxford: Oxford University Press. 2006.

18. Lo B, Ruston D, Kates L, Arnold R, Cohen C, Faber-Langendoen $\mathrm{K}$, et al. Discussing Religious and Spiritual Issues at the End of Life. A Practical Guide for Physicians. JAMA 2002; 287 (6): 749-54.

19. Nolan S, Saltmarsh P, Leget C. Spiritual care in palliative care: Working towards an EAPC Task Force. Eur J Palliat Care 2011; 18: 86-9.

20. Benito E, Olivera A, Galiana L, Barreto P, Pascual A, Gomisc C, Barbero J. Development and validation of a new tool for the assessment and spiritual care of palliative care patients. J Pain Symptom Manage 2014. 47; 2: 1008-18.

21. Benito E, Barbero J, Payas A. (Eds). El acompañamiento espiritual en Cuidados Paliativos. Una introducción y una propuesta. Madrid: Aran-SECPAL, 2008.

22. Gobierno de Chile, Ministerio de Salud, Subsecretaría de Salud Pública. Ley 20.584. Regula los Derechos y Deberes que tienen las personas en relación con su Atención de Salud. 2012. Disponible en: http://www.leychile. $\mathrm{cl} / \mathrm{N} ? \mathrm{i}=1039348 \& \mathrm{f}=2012-10-01 \& \mathrm{p}=$

23. Speck P. Spiritual issues in palliative care. En: Doyle D, Hanks G \& MacDonald N (Eds.), Oxford Textbook of Palliative Medicine (2nd). Oxford, U.K.: Oxford University Press; 2003. p. 805-14.

24. Peterman A, Reeve C, Winford E, Salsman J, Tsevat J, Cotton S, et al. Measuring Meaning and Peace With the FACIT-Spiritual Well-Being Scale: Distinction Without a Difference? Psychol Assess. 2014; 26 (1): 127-37.

25. O'Connell K, Skevington S. Reviewing the assessment of spirituality and religion in health-related quality of life. Chronic Illn 2007; 3 (1): 77-87.

26. Becker N, Nakashima M, Canda E. Spiritual assessment 
in aging: a framework for clinicians. Gerontol Soc Work 2007; 48 (3): 331-47.

27. Whitford H, Olver I, Peterson M. Spirituality as a core domain in assessment of quality of life in oncology. Psychooncology 2008; 17 (11): 1121-8.

28. Kreitzer M, Gross C, Waleekhachonloet O, Reilly-Spong M, Byrd M. The Brief Serenity Scale: A Psychometric Analysis of a Measure of Spirituality and Well-Being. J Holist Nurs 2009; 27 (1): 7-16.

29. Yong J, Kim J, Han SS, Puchalski CM. Development and validation of a scale assessing spiritual needs for korean patients with cancer. Journal of Palliative Care 2008; 24 (4): 240-6.

30. Carvajal A, Centeno C, Watson R, Martínez M, Sanz Rubiales A. ¿Cómo validar un instrumento de medida de la salud? An. Sist. Sanit. Navar 2011; 34 (1): 63-72.

31. Taboada P (Ed): Sedation at the end-of-life. An interdisciplinary approach. Dordrecht, The Netherlands: Springer Academic Publishers; 2015.

32. Pacomino L. Diccionario Teológico Enciclopédico. Navarra, España: Editorial Verbo Divino; 1980.
33. Laplanche J, Pontalis J. Diccionario de Psicoanálisis. Buenos Aires, Argentina: Paidós; 2004.

34. Emanuel E. ¿Qué hace que la investigación clínica sea ética? Siete requisitos éticos. En: Pellegrini A, Macklin $\mathrm{R}$, eds. Investigación en Sujetos Humanos: Experiencia Internacional. Santiago, Chile: Programa Regional de Bioética OPS/ OMS; 1999: 33-46.

35. Inouye $\mathrm{S}$. The confusion assessment method. Ann Inter Med 1990; 113: 941-8.

36. Quiroga P, Albalia C, Klaasen G. Validación de un test de tamizaje para el diagnóstico de demencia asociada a edad, en Chile. Rev Med Chile 2004; 132 (4): 467-78.

37. Freeman DH. Applied categorical data analysis. New York: Marcel Dekker Inc; 1987.

38. Cronbach LJ. Coefficient alpha and the internal structure of the tests. Psychometrika 1951; 297-334.

39. Cortina JM. What is Coefficient Alpha. An examination of theory and applications. Applied psychology 1993; 78: 98-104.

40. Feldt L, Woodruff D, Salih F. Statistical inference for Coefficient Alpha. Applied Psychologival Measurement 1987; 11: 93-103. 